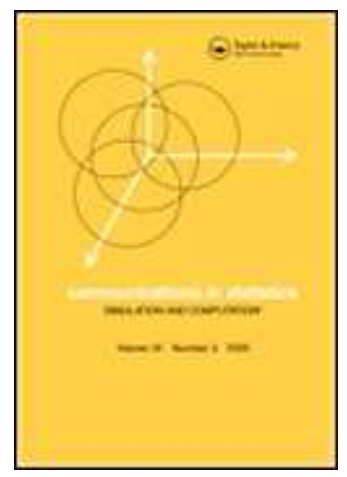

\title{
An adaptive test for the two-sample location problem based on U-statistics
}

\begin{tabular}{|c|c|}
\hline Journal: & Communications in Statistics - Simulation and Computation \\
\hline Manuscript ID: & LSSP-2007-0093.R2 \\
\hline Manuscript Type: & Original Paper \\
\hline $\begin{array}{r}\text { Date Submitted by the } \\
\text { Author: }\end{array}$ & 17-Dec-2007 \\
\hline Complete List of Authors: & $\begin{array}{l}\text { Koessler, Wolfgang; Humboldt-Universitaet zu Berlin, Informatik } \\
\text { Kumar, Narinder; Panjab University Chandigarh, Statistics }\end{array}$ \\
\hline Keywords: & $\begin{array}{l}\text { Wilcoxon-Mann-Whitney test, , asymptotic effcacy, asymptotic } \\
\text { power, simulation study, tailweight, skewness }\end{array}$ \\
\hline Abstract: & $\begin{array}{l}\text { For the two-sample location problem we consider a general class of } \\
\text { tests, all members of it are based on U-statistics. The asymptotic } \\
\text { efficacies are investigated in detail. } \\
\text { We construct an adaptive test where all statistics involved are } \\
\text { suitably chosen U-statistics. } \\
\text { It is shown that the proposed adaptive test has good asymptotic } \\
\text { and finite sample power properties. }\end{array}$ \\
\hline \multicolumn{2}{|c|}{$\begin{array}{l}\text { Note: The following files were submitted by the author for peer review, but cannot be converted } \\
\text { to PDF. You must view these files (e.g. movies) online. }\end{array}$} \\
\hline $\begin{array}{l}\text { koessler.tex } \\
\text { Figures_KoesslerKumar.zip }\end{array}$ & \\
\hline
\end{tabular}

\section{S) ScholaroNE \\ Manuscript Central}




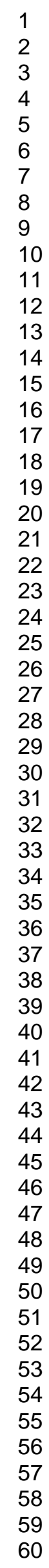

37 


\title{
An adaptive test for the two-sample location problem based on U-statistics
}

\author{
W. Kössler, Humboldt-Universität zu Berlin, \\ koessler@informatik.hu-berlin.de \\ N. Kumar, Panjab University Chandigarh, \\ nkumar67@yahoo.com
}

\begin{abstract}
For the two-sample location problem with continuous data we consider a general class of tests, all members of it are based on Ustatistics. The asymptotic efficacies are investigated in detail. We construct an adaptive test where all statistics involved are suitably chosen U-statistics. It is shown that the proposed adaptive test has good asymptotic and finite sample power properties.
\end{abstract}

Keywords: Wilcoxon-Mann-Whitney test, asymptotic efficacy, asymptotic power, simulation study, tailweight, skewness.

Subject classification: $62 \mathrm{G} 10,62 \mathrm{G} 20$

Corresponding author:

Wolfgang Kössler, Humboldt-Universität zu Berlin, Institut für Informatik Rudower Chaussee 25, D-12489 Berlin

Running title: Adaptive test based on U-statistics 


\section{Introduction}

Let $X_{1}, \ldots, X_{n_{1}}$ and $Y_{1}, \ldots, Y_{n_{2}}$ be independent random samples from populations with absolutely continuous distribution functions $F(x)$ and $F(x-\vartheta)$, $\vartheta \in \mathbf{R}$, respectively. We wish to test

$$
H_{0}: \quad \vartheta=0
$$

against

$$
H_{1}: \quad \vartheta>0 .
$$

The Wilcoxon-Mann-Whitney test is the most familiar nonparametric test for this problem. This test was generalised to linear rank tests with various other scores, such as the Median test, the normal scores test and the Savage test, see e.g. Hájek, Šidak and Sen (1999).

Another generalisation is to consider a class of tests based on U-statistics. This interesting class of tests has drawn considerable attraction in the literature (cf. e.g. Deshpande and Kochar (1982), Shetty and Govindarajulu (1988), Kumar (1997), Xie and Priebe (2000, 2002), John and Priebe (2007)).

Following Kumar, Singh and Öztürk (2003) a general class $U_{k, i}$ of Ustatistics is defined in Section 2. Local alternatives of the form $\vartheta=\theta_{N}=$ $\theta / \sqrt{N}, N=n_{1}+n_{2}$, are considered and the asymptotic efficacies of the tests based on $U_{k, i}$ are compared in Section 3. It is shown that there are different tests of this type which are efficient for densities with short, medium or long (right or left) tails, respectively. For example, the test based on $U_{5,1}$ is efficient for densities with short tails, and that based on $U_{5,3}$ is efficient for densities with long tails. However, the practising statistician has generally no clear idea on the underlying density, thus he/she should apply an adaptive test which takes into account the given data set. In Section 4 two versions of such an adaptive test are proposed, one of them is distribution-free. The adaptive tests first classify the underlying distribution with respect to some measures like (right and left) tailweight and skewness and then select an appropriate test based on U-statistics. Our adaptive test is compared briefly with adaptive tests based on linear rank tests in Section 5 and with the $t$ test and the Mann-Whitney-Wilcoxon test in Section 6 . In Section 7 a simulation study is performed and the finite sample power is compared with the asymptotic power. It is shown that one of the adaptive tests behaves well also for moderate sample sizes. A data example is provided in Section 8. We give some conclusions in Section 9. 


\section{Test statistics}

We consider the class of U-statistics, which was proposed by Kumar, Singh and Öztürk (2003). Let $k, 1 \leq k \leq \min \left(n_{1}, n_{2}\right)$, and $i, i \leq \frac{k+1}{2}$, be fixed integers. Define

$\Phi_{i}\left(x_{1}, \ldots, x_{k}, y_{1}, \ldots, y_{k}\right)= \begin{cases}2 & \text { if } x_{(i) k}<y_{(i) k} \text { and } x_{(k-i+1) k}<y_{(k-i+1) k} \\ 1 & \text { if either } x_{(i) k}<y_{(i) k} \text { or } x_{(k-i+1) k}<y_{(k-i+1) k} \\ 0 & \text { otherwise, }\end{cases}$

where $x_{(i) k}$ is the $i$ th order statistic in a subsample of size $k$ from the $X$ sample (and likewise for $y$ 's). Let $U_{k, i}$ be the U-statistic associated with kernel $\Phi_{i}$, i.e.

$$
U_{k, i}=\frac{n_{1} n_{2}}{\left(\begin{array}{c}
n_{1} \\
k
\end{array}\right) \cdot\left(\begin{array}{c}
n_{2} \\
k
\end{array}\right)} \sum \Phi_{i}\left(X_{r_{1}}, \ldots, X_{r_{k}}, Y_{s_{1}}, \ldots, Y_{s_{k}}\right)-n_{1} n_{2},
$$

where the summation extends over all possible combinations $\left(r_{1}, \ldots, r_{k}\right)$ of $k$ integers from $\left\{1, \ldots, n_{1}\right\}$ and all possible combinations $\left(s_{1}, \ldots, s_{k}\right)$ of $k$ integers from $\left\{1, \ldots, n_{2}\right\}$. The null hypothesis $H_{0}$ is rejected in favour of $H_{1}$ for large values of $U_{k, i}$.

Remark: The following special cases are of particular interest.

For $i=1$ and $k=1$ we have the Wilcoxon-Mann-Whitney test.

For $i=1$ or $i=k$ we have the Deshpande-Kochar test (cf. Deshpande and Kochar, 1982).

For $i=(k+1) / 2$ we have the Kumar-test (cf. Kumar, 1997).

Let

$$
\begin{aligned}
\varphi_{1,0}^{(i)}(x) & =\mathbf{E} \Phi_{i}\left(x, X_{2}, \ldots, X_{k}, Y_{1}, \ldots, Y_{k}\right) \\
\varphi_{0,1}^{(i)}(y) & =\mathbf{E} \Phi_{i}\left(X_{1}, \ldots, X_{k}, y, Y_{2}, \ldots, Y_{k}\right) \\
\zeta_{1,0}^{(i)} & =\operatorname{Var}\left(\varphi_{1,0}^{(i)}(X)\right) \\
\zeta_{0,1}^{(i)} & =\operatorname{Var}\left(\varphi_{0,1}^{(i)}(Y)\right),
\end{aligned}
$$

where $\mathbf{E}$ and Var denote the expectation and variance respectively. Moreover, let $F_{(i) k}($.$) be the cumulative distribution function of the i$ th order statistics of a sample of size $k$. 
Proposition 2.1 (cf. Kumar, Singh and Öztürk, 2003) Under assumptions $N \rightarrow \infty, n_{1} / N \rightarrow \lambda, 0<\lambda<1$ the limiting distribution of $N^{1 / 2}\left(U_{k, i}-\right.$ $\left.\eta_{k, i}\right) / \sigma_{k, i}$ is standard normal, where expectation $\eta_{k, i}=\mathbf{E} U_{k, i}$ and variance $\sigma_{k, i}^{2}=\operatorname{Var}\left(U_{k, i}\right)$ have the forms

$$
\begin{aligned}
\eta_{k, i} & =n_{1} n_{2}\left(\int_{-\infty}^{\infty} F_{(i) k}(y) d F_{(i) k}(y-\theta)+\int_{-\infty}^{\infty} F_{(k-i+1) k}(y) d F_{(k-i+1) k}(y-\theta)\right)-n_{1} n_{2} \\
\sigma_{k, i}^{2} & =n_{1}^{2} n_{2}^{2}\left(\frac{k^{2} \zeta_{10}^{(i)}}{\lambda}+\frac{k^{2} \zeta_{01}^{(i)}}{1-\lambda}\right) .
\end{aligned}
$$

Remark: Under $H_{0}$ we have $\eta_{k, i}=0$ and

$$
\sigma_{k, i}^{2}=n_{1}^{2} n_{2}^{2} k^{2} \frac{\rho_{k, i}}{\lambda(1-\lambda)}
$$

where $\rho_{k, i}$ depends on $k$ and $i$ only. The expression for $\rho_{k, i}$ is rather long, that is why we do not write it out. It can be found in Kumar, Singh and Öztürk (2003), pp.125-126.

\section{The asymptotic efficacies}

The asymptotic efficacies $\mathrm{AE}$ of the statistics $U_{k, i}$ under the alternative $\theta_{N}=$ $N^{-1 / 2} \cdot \theta$ are given by

$$
A E\left(U_{k, i} \mid f\right)=\lambda(1-\lambda) \cdot C_{k, i}^{2}(f),
$$

where $f(\cdot)$ denotes the probability density function belonging to the c.d.f. $F(\cdot)$ and

$$
\begin{array}{r}
C_{k, i}(f)=\frac{\left(\left(\begin{array}{c}
k \\
i
\end{array}\right) i\right)^{2}}{\left(k^{2} \rho_{k, i}\right)^{1 / 2}} \cdot\left(\int_{-\infty}^{\infty}(F(x))^{2 i-2}(1-F(x))^{2 k-2 i} f^{2}(x) d x+\right. \\
\left.\int_{-\infty}^{\infty}(F(x))^{2 k-2 i}(1-F(x))^{2 i-2} f^{2}(x) d x\right)
\end{array}
$$

(cf. Kumar, Singh and Öztürk, 2003).

Note that the asymptotic efficacy is defined by the limit of $\eta_{k, i}^{2} / \sigma_{k, i}^{2}$, cf. Noether (1955). 
From Kumar, Singh and Öztürk (2003) we know that for some underlying distributions the test based on $U_{k, i}$ has highest efficacy if $k$ is as large as possible as long as it is less than sample size. For example, for the uniform $i$ should be 1 or $k$, and for the double exponential $k$ should be odd and $i=(k+1) / 2$. Therefore, for further investigations we have to make some restrictions. We restricted the choice of $k$ as $k \leq 5$ ourselves just for illustrative purpose. Of course, computations can be made for values of $k$ beyond that. We compute the asymptotic Pitman efficacies for all tests $U_{k, i}$ with $1 \leq i \leq k, k \leq 5$. Values of the factors $C_{k, i}^{2}(f)$ for various densities are presented in Table 1. We also give the corresponding factors for the $t$-test (see the first column). The L-DE density was proposed by Policello and Hettmansperger (1976), the U-L by Gastwirth (1965), the RST is named after Ramberg, Schmeiser and Tukey, cf. Ramberg and Schmeiser (1972, 1974), CN $(\epsilon, \sigma)$ is the scale contaminated normal with contaminating proportion $\epsilon$, Mielke denotes the Mielke (1972) density and BT is the density of Box and Tiao (1962).

The bold entries denote, for the given density, the asymptotically best test among the considered tests. (If the $t$-test is the asymptotically best, it is denoted in italics. Note that the $t$-test is not allowed to be included in the adaptive procedure since the adaptive test requires independence between the two steps, see Proposition 4.1 below.) On the first view we see that the columns for $U_{3,1}, U_{5,1}$ and $U_{5,3}$ have the most bold entries. This observation gives rise to the idea to base an adaptive test on these few statistics. (The classical test $U_{1,1}$ is also included in the adaptive test.)

To get a closer idea how to classify densities we apply the method of Hall and Joiner (1982). The content of information in the asymptotic efficacy matrix is analysed by a principal component analysis where the densities are the observations and the efficacies of the $U_{i, j}$ are the variables. The first principal component explains already $96 \%$ of the variability (Figure 1). For better visibility we display the values of the first two (varimax rotated) principal components (Factor 1 and Factor 2). In Figure 1 nearly symmetric densities with short tails are denoted by a green plus, that with short-medium and long-medium tails by a cyan X and a blue star, respectively, and that with long tails with a red dot. Skew densities are denoted by a black plus (short tails) and a yellow dot (medium tails) respectively. The dots denote densities with long tails, the stars and $\mathrm{X}$ that with medium tails, and the 
plus that with short tails. On the left side we have densities with long tails, in the centre that with medium tails, and on the right that with short tails. For an exact definition what we understand by long, medium and short tails see below. On the first view we see that the $A E\left(U_{k, i}\right)$ classify the densities according to their tailweight. The skewness seems to play a marginal role only.

\section{Insert Figure 1 about here}

\section{Adaptive test}

There are some proposals for adaptive tests for the two-sample location problem in the literature, see e.g. Hogg $(1974,1982)$, Hogg, Fisher and Randles (1975), Ruberg (1986) and Büning (1994). We apply the concept of Hogg (1974), that is, to classify at first the type of the underlying density with respect to one measure of skewness $\hat{s}$ and to three measures of tailweight $\hat{t}$, $\hat{t}_{r}$ and $\hat{t}_{l}$, which are defined by

$$
\begin{aligned}
\hat{s} & =\frac{\hat{Q}(0.95)+\hat{Q}(0.05)-2 \cdot \hat{Q}(0.5)}{\hat{Q}(0.95)-\hat{Q}(0.05)} \\
\hat{t} & =\frac{\hat{Q}(0.95)-\hat{Q}(0.05)}{\hat{Q}(0.85)-\hat{Q}(0.15)} \\
\hat{t}_{l} & =\frac{\hat{Q}(0.5)-\hat{Q}(0.05)}{\hat{Q}(0.5)-\hat{Q}(0.15)} \quad \hat{t}_{r}=\frac{\hat{Q}(0.95)-\hat{Q}(0.5)}{\hat{Q}(0.85)-\hat{Q}(0.5)}
\end{aligned}
$$

where $\hat{Q}(u)$ is the so-called classical quantile estimate of $F^{-1}(u)$,

$$
\hat{Q}(u)=\left\{\begin{array}{lll}
X_{(1)}-(1-\delta) \cdot\left(X_{(2)}-X_{(1)}\right) & \text { if } u<1 /(2 \cdot N) \\
(1-\delta) \cdot X_{(j)}+\delta \cdot X_{(j+1)} & \text { if } \quad \frac{1}{2 \cdot N} \leq u \leq \frac{2 \cdot N-1}{2 \cdot N} \\
X_{(N)}+\delta\left(X_{(N)}-X_{(N-1)}\right) & \text { if } u>(2 \cdot N-1) /(2 \cdot N),
\end{array}\right.
$$

where $\delta=N \cdot u+1 / 2-j$ and $j=\lfloor N \cdot u+1 / 2\rfloor$. Note that $\hat{t}_{l}$ and $\hat{t}_{r}$ are measures of left tailweight and right tailweight, respectively. All the measures are estimated from the pooled sample. 
In Tables 2 and 3 the values of the corresponding theoretical measures $s, t, t_{r}$ and $t_{l}$, for various selected densities are presented. (For symmetric densities we have $s=0$ and $t=t_{r}=t_{l}$.)

Comparing Table 1 with Tables 2 and 3 roughly we see that $U_{5,1}$ is the asymptotically best test for symmetric densities with small tailweight, $U_{3,1}$ for symmetric densities with small to medium tailweight, $U_{1,1}$ for symmetric densities with medium to larger tailweight, and $U_{5,3}$ for symmetric densities with large tailweight. The tests $U_{3,1}$ and $U_{1,1}$ should be included in an adaptive test since they are the (asymptotically) best for the normal and for the logistic density, respectively (at least among the considered tests).

The measure of skewness gives no clear classification idea. That is why we consider left tailweight $t_{l}$ and right tailweight $t_{r}$, and classify densities as densities with partially short tails if $t_{l}<1.55$ or $t_{r}<1.55$. They are classified to have partially medium tails if $t_{l}<1.8$ or $t_{r}<1.8$ and if they have not partially short tails.

The reasoning of the last two sections gives rise to the following adaptive test.

Define regions $E_{1}, \ldots, E_{7}$ of $\mathbb{R}^{4}$ which are based on the so called selector statistic $\hat{S}=\left(\hat{s}, \hat{t}, \hat{t}_{l}, \hat{t}_{r}\right)$

$$
\begin{aligned}
& E_{1}=\{\hat{t}<1.55,|\hat{s}| \leq 0.2\} \quad \text { "nearly symmetric, short tails" } \\
& E_{2}=\{1.55 \leq \hat{t}<1.65,|\hat{s}| \leq 0.2\} \quad \text { "nearly symmetric, light medium tails" } \\
& E_{3}=\{1.65 \leq \hat{t} \leq 1.8,|\hat{s}| \leq 0.2\} \quad \text { "nearly symmetric, heavy medium tails" } \\
& E_{4}=\{\hat{t}>1.8,|\hat{s}| \leq 0.2\} \quad \text { "nearly symmetric, long tails" } \\
& E_{5}=\left\{\left(\hat{t}_{l}<1.55 \vee \hat{t}_{r}<1.55\right),|\hat{s}|>0.2\right\} \quad \text { "skew, partially short tails" } \\
& E_{6}=\left\{\left(\hat{t}_{l}>1.65 \wedge \hat{t}_{r}>1.65\right),|\hat{s}|>0.2\right\} \quad \text { "skew, long tails" } \\
& E_{7}=\left\{\hat{t}_{l} \geq 1.55, \hat{t}_{r} \geq 1.55,|\hat{s}|>0.2\right\} \backslash E_{6} \quad \text { "skew, partially medium tails" }
\end{aligned}
$$

where $\hat{s}, \hat{t}, \hat{t}_{l}$ and $\hat{t}_{r}$ are given by (1) to (3). Note that there was no density which belongs to class $E_{7}$.

The cutoff values of the regions are determined in such a way that the vast majority of densities is classified correctly, i.e. they fall in the class that has the highest asymptotic power (cf. Tables 2 and 3 with Table 1). For example, the normal (tailweight $t=1.59$, cf. Table 2 ) is classified to $E_{2}$, and the test $U_{3,1}$, which is the best among the considered tests (cf. Table 1), is performed. The logistic is mapped to region $E_{3}$ and the optimal test $U_{1,1}$ is performed. Similar observations for the other densities lead to the given cutoff values. In few cases, if the classification is not correct, then the efficacy loss is very small in almost all cases. In Table 1 the chosen test is underlined 
if it is not already the (bold) best.

Now, we propose the Adaptive test $A$ which is based on the four Ustatistics $U_{5,1}, U_{3,1}, U_{1,1}$, and $U_{5,3}$. We denote the tests by $(5,1),(3,1),(1,1)$ and $(5,3)$, respectively.

$$
A=A(\hat{S})= \begin{cases}(5,1) & \text { if } \quad \hat{S} \in E_{1} \cup E_{5} \\ (3,1) & \text { if } \quad \hat{S} \in E_{2} \cup E_{7} \\ (1,1) & \text { if } \quad \hat{S} \in E_{3} \\ (5,3) & \text { if } \quad \hat{S} \in E_{4} \cup E_{6}\end{cases}
$$

In Figure 2 the corresponding adaptive scheme is given. As indicated above the skewness plays only a marginal role. It is included in the adaptive scheme implicitly by left and right tailweight.

\section{Insert Figure 2 about here}

The two-stage procedure defined above is asymptotically distribution-free since the selector statistic $\hat{S}$ is based on the order statistic only and the Ustatistics are based on the ranks only.

The Adaptive test A is only asymptotically distribution-free because asymptotic critical values are used in the adaptive scheme.

Proposition 4.1 Let $\sigma_{F}$ be the standard deviation of the underlying $c d f . F$, if it exists and let $\left\{\theta_{N}\right\}$ be a sequence of 'near' alternatives with $\sqrt{N} \theta_{N} \rightarrow$ $\sigma_{F} \theta$. The asymptotic power function of the Adaptive test $A$ equals

$$
\beta(\theta)= \begin{cases}1-\Phi\left(z_{1-\alpha}-\sqrt{A E\left(U_{5,1} \mid f\right)} \cdot \sigma_{F} \cdot \theta\right) & \text { if } f \in E_{1} \cup E_{5} \\ 1-\Phi\left(z_{1-\alpha}-\sqrt{A E\left(U_{3,1} \mid f\right)} \cdot \sigma_{F} \cdot \theta\right) & \text { if } f \in E_{2} \cup E_{7} \\ 1-\Phi\left(z_{1-\alpha}-\sqrt{A E\left(U_{1,1} \mid f\right)} \cdot \sigma_{F} \cdot \theta\right) & \text { if } f \in E_{3} \\ 1-\Phi\left(z_{1-\alpha}-\sqrt{A E\left(U_{5,3} \mid f\right)} \cdot \sigma_{F} \cdot \theta\right) & \text { if } f \in E_{4} \cup E_{6}\end{cases}
$$

Proof. Let be $h=1$ if $(k, i)=(5,1), h=2$ if $(k, i)=(3,1), h=3$ if $(k, i)=(1,1), h=4$ if $(k, i)=(5,3)$ Let be $T_{1}=U_{5,1}, T_{2}=U_{3,1}, T_{3}=U_{1,1}$, $T_{4}=U_{5,3}$ and $D_{1}=E_{1} \cup E_{5}, D_{2}=E_{2} \cup E_{7}, D_{3}=E_{3}, D_{4}=E_{4} \cup E_{6}$. The proposition follows from the total probability theorem and from the 
consistency of the selector statistics, i.e.

$$
\begin{aligned}
\beta(\theta) & =\sum_{h=1}^{4} P_{\sigma_{F} \theta}\left(T_{h}>c_{\alpha h} \mid T_{h} \text { chosen }\right) \cdot P_{\sigma_{F} \theta}\left(T_{h} \text { chosen }\right) \\
& =\sum_{h=1}^{4}\left(1-\Phi\left(z_{1-\alpha}-\sqrt{A E\left(T_{h} \mid f\right)} \cdot \sigma_{F} \cdot \theta\right)+o(1)\right) \cdot\left\{\begin{array}{l}
1+o(1) \quad \text { if } f \in D_{h} \\
o(1) \quad \text { else }
\end{array}\right. \\
& \sim 1-\Phi\left(z_{1-\alpha}-\sqrt{A E\left(T_{h} \mid f\right)} \cdot \sigma_{F} \cdot \theta\right) \quad \text { if } \quad f \in D_{h},
\end{aligned}
$$

where $c_{\alpha h}$ is the $(1-\alpha)$-quantile of the asymptotic null distribution of $T_{h}$.

Remark: The factor $\sigma_{F}$ is introduced to have similar power values for the various distributions. For the Cauchy the factor $\sigma_{F}$ is set to $\sigma_{F}=\sigma_{C a u}=$ $F^{-1}(\Phi(1))=1.8373$.

The Adaptive test $A(\hat{S})$ is based on selector statistics computed from the pooled sample. However, location differences may effect the estimates of tailweight and skewness. That is why we consider also a modification $A\left(\hat{S}^{*}\right)$ of the adaptive test, where tailweights and skewness are estimated from the single samples. Let $\hat{s}_{i}$ and $\hat{t}_{i}, \hat{t}_{l, i}$ and $\hat{t}_{r, i}, i=1,2$ be statistics of the form (1) to (3) for skewness and tailweight, left tailweight and right tailweight, respectively. Applying the $A\left(\hat{S}^{*}\right)$-test the selector statistic $\hat{S}^{*}=\left(\hat{s}^{*}, \hat{t}^{*}, \hat{t}_{l}^{*}, \hat{t}_{r}^{*}\right)$ with

$$
\begin{aligned}
\hat{s}^{*}=\frac{n_{1}}{N} \hat{s}_{1}+\frac{n_{2}}{N} \hat{s}_{2} & \hat{t}^{*} & =\frac{n_{1}}{N} \hat{t}_{1}+\frac{n_{2}}{N} \hat{t}_{2} \\
\hat{t}_{l}^{*}=\frac{n_{1}}{N} \hat{t}_{l, 1}+\frac{n_{2}}{N} \hat{t}_{l, 2} & \hat{t}_{r}^{*} & =\frac{n_{1}}{N} \hat{t}_{r, 1}+\frac{n_{2}}{N} \hat{t}_{r, 2}
\end{aligned}
$$

is used instead of $\hat{s}, \hat{t}, \hat{t_{l}}$ and $\hat{t_{r}}$. This procedure is also asymptotically distribution-free. However, it is not distribution-free also if the exact critical values are used. This property is due to the fact that the selector statistic is no longer based on the pure order statistic.

For various densities asymptotic power functions (together with finite power functions) are given in Figures 3 and 4.

The blue dotted line is for $U_{1,1}$, the violet short-dashed line for $U_{3,1}$, the green long-dashed line for $U_{5,1}$, the red dashed-dotted line for $U_{5,3}$ (and the black continuous line for the adaptive test).

Remark: Another adaptive test which is based on U-statistics, but with another classes of U-statistics (and in another context) is proposed in Kössler 
(2005). It turns out, that the adaptive test presented here is slightly better for the majority of considered densities, especially for short tail densities (uniform, exponential).

Remark: Another kind of (nonrestrictive) adaptive tests was constructed by John and Priebe (2007). They take the class of weighted generalised Mann-Whitney-Wilcoxon (WGMWW) statistics proposed by Xie and Priebe (2002), and estimate in a first step the asymptotic efficacies, where the weights are considered as parameters. In a second step these weights are determined in such a way that the estimated asymptotic efficacy is maximised. Then the test based on the resulting WGMWW statistic is performed.

\section{Comparison to adaptive tests based on lin- ear rank statistics}

Restrictive adaptive tests for the two-sample location problem based on linear rank tests are proposed by Hogg $(1974,1982)$, Hogg, Fisher and Randles (1975), Handl (1986) and Büning (1994). All of them are based on the concept of Hogg (1974), and they use few linear rank statistics, with the following scores: Gastwirth (GA, for short tails), Wilcoxon (WI, for medium tails), Median (Hogg, 1974, and Hogg et al., 1975, Long-tail (LT, Handl, 1986) and Büning, 1994, both for long tails) and Hogg-Fisher-Randles (HFR, for right-skew densities). Since the Median test is known to be bad for most densities (except for the doubleexponential), we restrict to the scores GA, WI, LT and HFR, and call the corresponding adaptive scheme $B(\hat{S})$ (where the form of $\hat{S}$ is not of interest here). For some densities we take the respective two asymptotically best tests in the the Adaptive schemes $A(\hat{S})$ and $B(\hat{S})$ (cf. Table 4).

For the classical densities considered the U-statistics based test $A(\hat{S})$ has slightly higher asymptotic power than the test $B(\hat{S})$. (For the logistic they are, of course, the same.) For the densities U-L (0.75) and L-D (0.75) that are 'optimal' densities for the tests GA and LT (cf. Büning and Kössler, 1999) it is vice versa. 


\section{Comparison to the $t$-test and to the Wilcoxon test}

To evaluate the adaptive test we compare the $\mathrm{AE}$ values for the $t$-test, the Wilcoxon test, and for the Adaptive test $\mathrm{A}(\mathrm{S})$. The test with the highest $\mathrm{AE}$ gets rank one, that with the second highest rank two, and that with the lowest $\mathrm{AE}$ (among the three tests) rank three, all for the various densities considered. In the case of equal AE values, average ranks are taken. The results are presented in Table 5. The avarage rank over all 86 densities is 1.23 for the Adaptive test $\mathrm{A}(\mathrm{S})$, in comparison to 2.10 for the Wilcoxon test and 2.67 for the $t$-test. Note that this result is not surprising since the adaptive test $A(S)$ is constructed in such a way that it has high AE.

\section{Simulation study}

In order to assess whether the asymptotic theory can also be applied for medium to small sample sizes a simulation study (10,000 replications each) is performed. We choose the following six distributions:

- Uniform distribution (density with small tailweight),

- Normal distribution (density with medium tailweight),

- Logistic distribution (density with medium tailweight),

- Doubleexponential distribution (density with large tailweight),

- Cauchy distribution (density with very large tailweight),

- Exponential distribution (very skew density)

We consider the four single tests $U_{5,1}, U_{3,1}, U_{1,1}, U_{5,3}$ and the two Adaptive tests $A(\hat{S})$ and $A\left(\hat{S}^{*}\right)$. The sample sizes $n_{1}=n_{2}=10,20,40$ and the alternatives $\theta_{N}=N^{-1 / 2} \theta \sigma_{F}$ with various $\theta$ are considered. Recall that the factor $\sigma_{F}$ denotes the standard deviation of the underlying distribution function $F$ $\left(\sigma_{\text {Cau }}:=F^{-1}(\Phi(1))=1.8373\right)$.

The Adaptive test $A\left(\hat{S}^{*}\right)$ is slightly anticonservative, also for $n_{1}=n_{2}=$ 40, with correspondingly slightly higher power than the Adaptive test $A(\hat{S})$ (except for the Cauchy). This test is not considered here. 
For $n_{1}=n_{2}=10$ and $n_{1}=n_{2}=20$ almost all tests (except the test $U_{1,1}$ which attains the level of significance) are slightly anticonservative, for $n_{1}=n_{2}=10$ the attained level of significance is always less than 0.08 , and for $n_{1}=n_{2}=20$ it is less than 0.06 and therefore $\epsilon=0.2$-robust. (For the notion of $\epsilon$-robustness see e.g. Rasch, Teuscher, and Guiard (2007).) For $n_{1}=n_{2}=40$ the results of the simulation study are summarized in Figures 3 and 4. Again, the blue dotted line is for $U_{1,1}$, the violet short-dashed line for $U_{3,1}$, the green long-dashed line for $U_{5,1}$, the red dashed-dotted line for $U_{5,3}$ and the continuous line is for the Adaptive test $A(\hat{S})$. At first we see that, for $n_{1}=n_{2}=40$, the finite power is well approximated by the asymptotic power (except for the Cauchy). Moreover, it can be seen that, for a given density, there is always, sometimes together with another test, a single test which is the best. The test $U_{5,1}$ is the best for the uniform and for the exponential (together with the Adaptive test $A(\hat{S})$ ), the test $U_{1,1}$ is the best for the normal and for the logistic density, and the test $U_{5,3}$ is the best for the doubleexponential and for the Cauchy. All these facts are not surprising. Also, not surprisingly, the tests $U_{5,1}$ and $U_{5,3}$ may be bad for some densities. The tests $U_{1,1}$ and $A(\hat{S})$ are, over all densities, the best. However, for the uniform and for the exponential densities the adaptive test is clearly better.

For the Cauchy density, somewhat surprisingly, the test $U_{1,1}$ is clearly better than the adaptive tests. The Adaptive test $A\left(\hat{S}^{*}\right)$ is better than $A(\hat{S})$. The reason for these facts is, that for small and moderate sample sizes, the misclassification rate into the class $E_{1}$ is relatively large.

Insert Figures 3 and 4 about here

\section{Data example}

At the 50kms ski-running competition in the Jizerske hory 2002 altogether 2320 runners finished the race. We consider only two age groups, where the first group contains men with an age less then 40, and the second group men of an age of 40 to 49 . The (perhaps somewhat trivial?) conjecture is that the running times of the older are longer than that of the younger men. Figure 5 gives a box plot of these data. 
At first, we estimate skewness and tailweight, cf. (1) and (2), and we obtain $\hat{s}=0.116$ and $\hat{t}=\mathbf{1 . 5 0 0}$. (The computation of left and right tailweights is not necessary here.) According to our adaptive procedure the test $U_{5,1}$ is chosen. We obtain $U_{5,1}=1.633$ which results in an one-sided p-value of 0.0511. Although the boxes look very similar we obtain a relatively small p-value. On one hand this fact may be due to the large sample sizes, on the other hand our procedure gives a weak evidence against $H_{0}$. For comparison we also computed the one-sided p-values for the (here not recommended) Mann-Whitney Wilcoxon test, $p=0.181$ and for the $t$-test, $p=0.188$.

\section{Conclusions}

What are the results of our study? At first, we see that the finite power of the considered tests based on U-statistics can be well approximated by their asymptotic power. Second, there are modifications of the "classical" MannWhitney test MW that may have (considerably) higher power than MW for symmetric as well asymmetric densities. Third, the Adaptive test $A(\hat{S})$ is a serious alternative for the Wilcoxon-Mann-Whitney test $U_{1,1}$ for moderate to large sample sizes. Especially, for short-tail densities and for skew densities the adaptive test considered here is better than the adaptive test $B(\hat{S})$ based on linear rank tests.

Many tests based on U-Statistics have good power properties. They can be applied to a broader class of underlying densities than a linear rank test with fixed scores.

For moderate to large sample sizes (about $n_{1}, n_{2} \geq 20$ ) the recommendation for the practising statistician is as follows: If the density is known to be nearly normal take the $t$-test. If it is only known that it has medium tails then take the Mann-Whitney-Wilcoxon test or the $t$-test. (For the robustness of the $t$-test see e.g. Büning (1991) or Rasch, Teuscher, and Guiard (2007) and the references therein.) For densities with short tails take the test $U_{5,1}$, and for those with long tails the test $U_{5,3}$. For a completely unknown density take the adaptive test $A(\hat{S})$. 
For relatively small sample sizes (about $n_{1}, n_{2}=10$ ) take the MannWhitney-Wilcoxon test. If it is known that the densities have not too large tails, then $t$-test may be applied. (Of course, the $t$-test is allowed only, if outliers can be excluded.)

Note that we considered the location problem for continuous data only. How the procedures behave for ordinal data may be a topic for further studies. Comparisons of the Mann-Whitney-Wilcoxon test with the $t$-test are made in the recent paper of Rasch, Teuscher, and Guiard (2007). Further investigations are also desirable for more general alternatives, such as $F \neq G$ or for the case of additional scale differences between $F$ and $G$.

Acknowledgements The authors are grateful to the editor and the referees who gave valuable hints on an improvement of an earlier version of this paper.

\section{References}

Box, G.E.P., Tiao, G.C. (1962). A further look at robustness via Bayes's theorem. Biometrika, 49:419-432.

Büning, H. (1991). Robuste und adaptive Tests. De Gruyter, Berlin.

Büning, H. (1994). Robust and adaptive tests for the two-sample location problem. OR Spektrum, 16:33-39.

Büning, H. and Kössler, W. (1999). The Asymptotic Power of Jonckheere-Type Tests for Ordered Alternatives, Australian \& NewZealand Journal of Statistics, vol.41:67-78.

Deshpande, J.V., Kochar, S.C. (1982). Some competitors of Wilcoxon-MannWhitney test for location alternatives. Journal of Indian Statistical Association, 19:9-18.

Gastwirth, J.L. (1965). Percentile modifications of two-sample rank tests. Journal of the American Statistical Association, 60:1127-1141.

Hájek, J., Šidák, Z., Sen, P.K. (1999). Theory of Rank Tests, San Diego: Academic Press.

Hall, D.L., Joiner, B.L. (1983). Asymptotic Relative Efficiency of $R$-estimators of Location. Communications in statistics, Theory and Methods, 12:739763 . 
Handl, A (1986). Masszahlen zur Klassifizierung von Verteilungen bei der Konstruktion adaptiver verteilungsfreier Tests im unverbundenen Zweistichprobenproblem, unpublished dissertation, Freie Universität Berlin.

Hogg, R.V. (1974). Adaptive robust procedures: partial review and some suggestions for future applications and theory. Journal of the American Statistical Association, 69:909-923.

Hogg, R.V. (1982). On adaptive statistical inference. Communications in Statistics, Theory and Methods, 11:2531-2542.

Hogg, R.V., Fisher, D.M., Randles, R.H. (1975). A two-sample adaptive distribution-free test. Journal of the American Statistical Association, 70:656-661.

John, M., Priebe, C.E. (2007). A data-adaptive methodology for finding an optimal weighted generalized Mann-Whitney-Wilcoxon statistic. Computational Statistics \& Data Analysis, 51:4337-4353.

Kössler, W. (2005). Some c-Sample Rank Tests of Homogeneity Against Ordered Alternatives based on U-statistics. Journal of Nonparametric Statistics, 17:777-796.

Kumar, N. (1997). A Class of Two-Sample tests for Location Based on SubSample Medians. Communications in Statistics, Theory and Methods, 26:943-951.

Kumar, N., Singh, R.S., Öztürk, Ö. (2003). A New Class of Distribution-Free Tests for Location parameters. Sequential Analysis, 22:107-128.

Mielke, P.W.Jr. (1972). Asymptotic behaviour of two-sample tests based on powers of ranks for detecting scale and location alternatives. Journal of the American Statistical Association, 67:850-854.

Noether, G.E. (1955). On a Theorem of Pitman. Annals of Mathematical Statistics, 26:64-68.

Policello, G.E., Hettmansperger, T.P. (1976). Adaptive robust procedures for the one-sample location problem. Journal of the American Statistical Association, 71:624-633.

Ramberg, J.S., Schmeiser, B.W. (1972). An approximate method for generating symmetric random variables, Communications of the ACM, 11:987990. 
Ramberg, J.S., Schmeiser, B.W. (1974). An approximate method for generating asymmetric random variables, Communications of the ACM, 17:78-82.

Rasch, D., Teuscher, F., and Guiard, V. (2007). How robust are tests for two independent samples? Journal of Statistical Planning and Inference, 137:2706-2720.

Ruberg, S.J. (1986). A continuously adaptive nonparametric two-sample test. Communications in Statistics, Theory and Methods, 15:2899-2920.

Shetty, I.D., Govindarajulu, Z. (1988). A Two-Sample Test for Location. Communications in Statistics, Theory and Methods, 27:2389-2401.

Xie, J., Priebe, C.E. (2000). Generalizing the Mann-Whitney-Wilcoxon Statistic. Nonparametric Statistics, 12:661-682.

Xie, J., Priebe, C.E. (2002). A weighted generalization of the Mann-WhitneyWilcoxon Statistic. Journal of Statistical Planning and Inference, 102:441466. 
Figure 1. The first two principal components

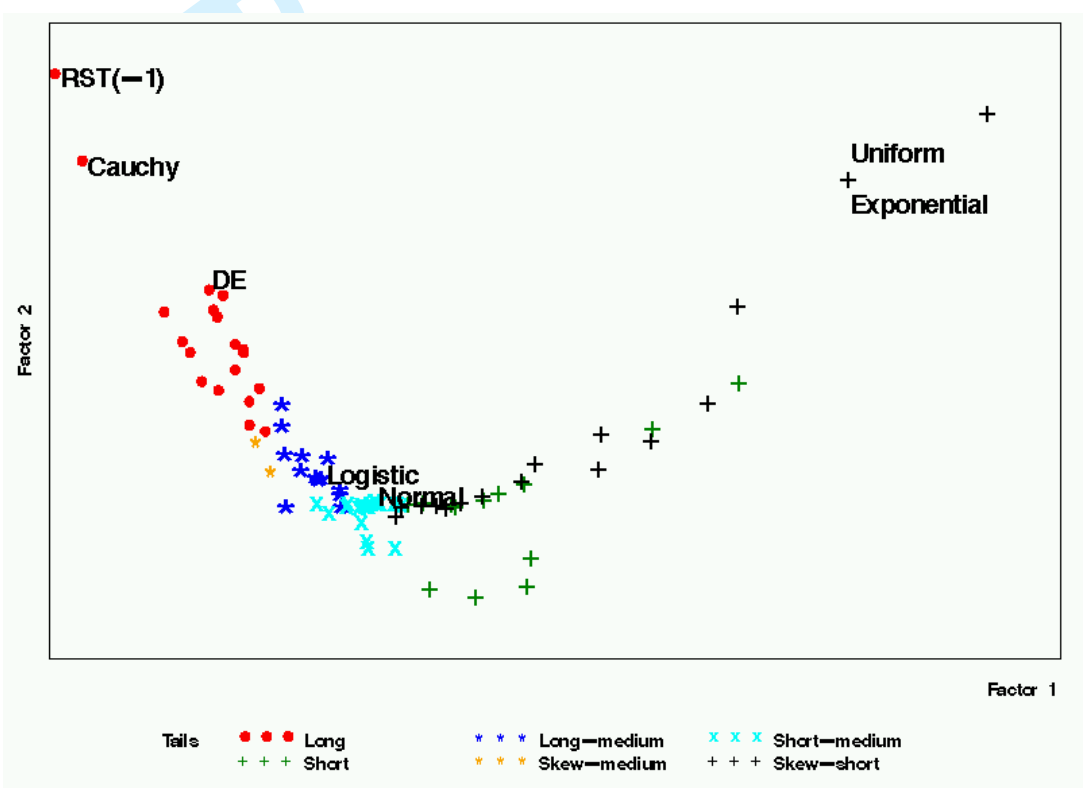


Figure 2. Adaptive scheme.

a) symmetric or moderately skew densities skewness

$|s|$

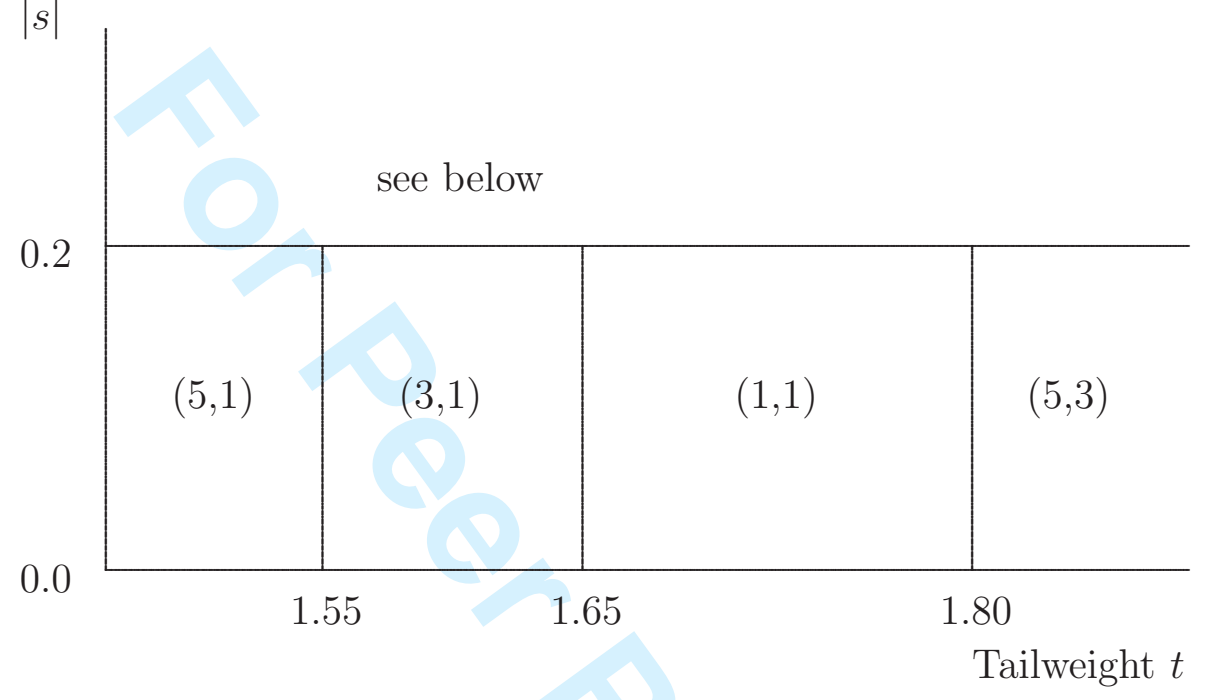

b) very skew densities $(|\hat{s}|>0.2)$

right tailweight

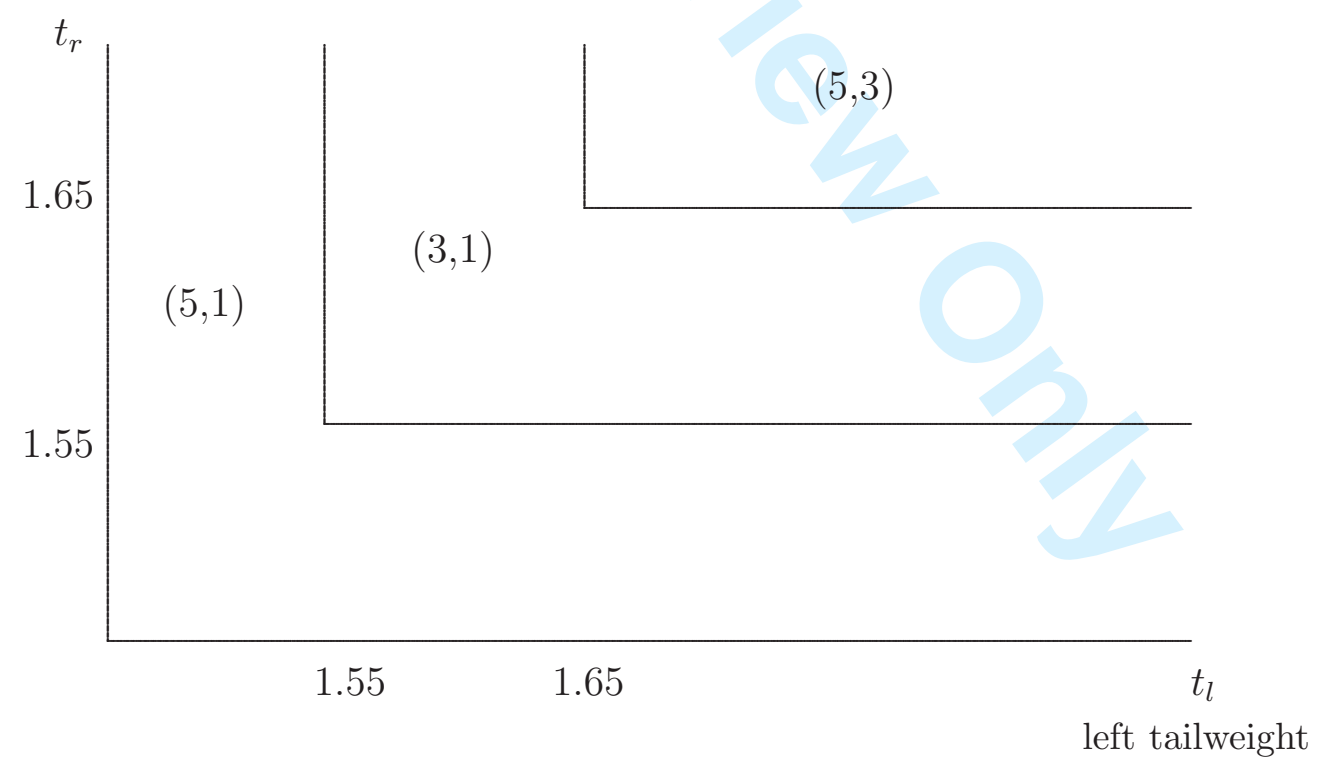


Figure 3. The asymptotic and finite $\left(n_{1}=n_{2}=40\right)$ power functions of the tests $U_{1,1}, U_{3,1}, U_{5,1}, U_{5,3}$ and $A(\hat{S})$; densities: uniform, normal and logistic.
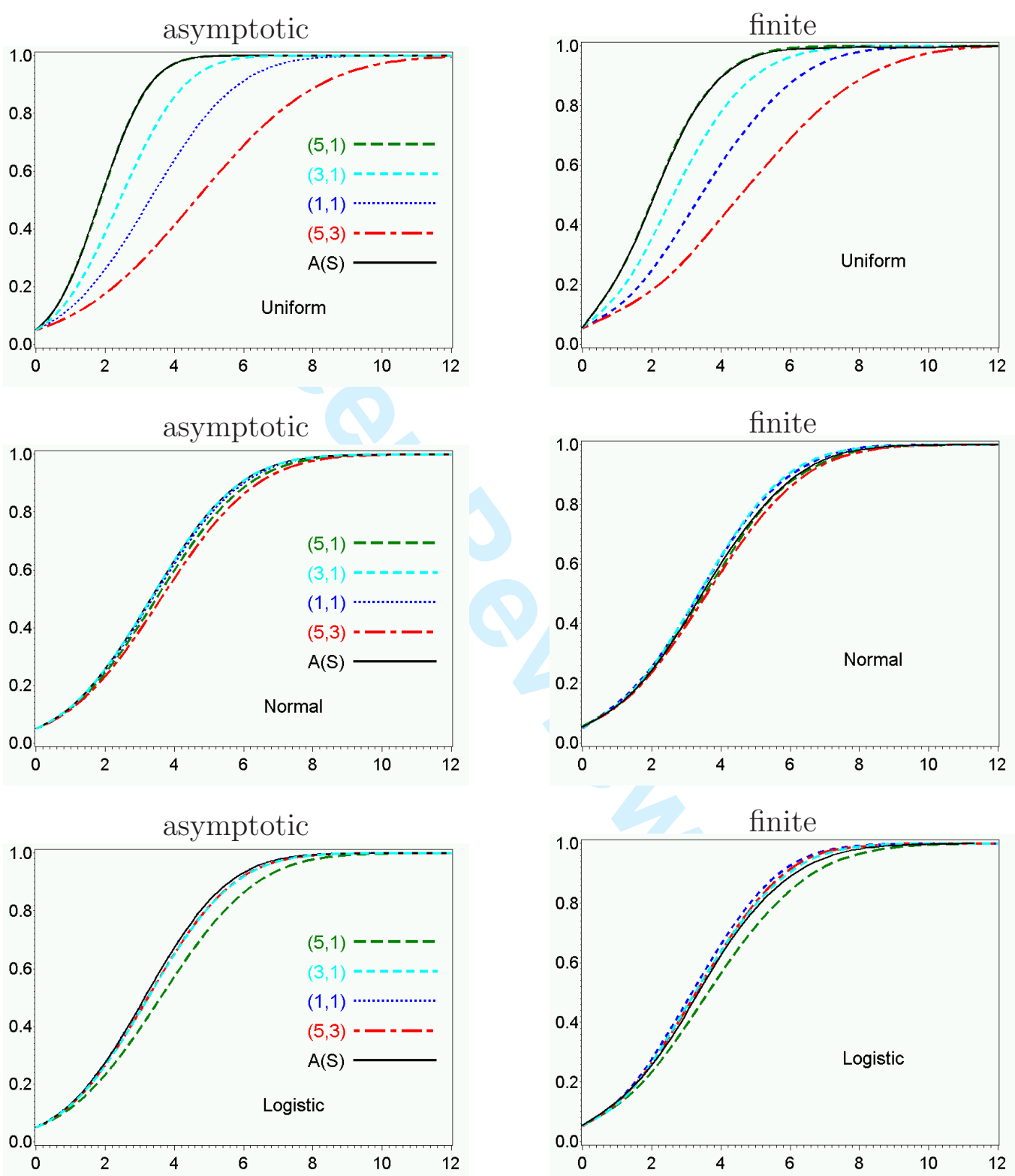
Figure 4. The asymptotic and finite $\left(n_{1}=n_{2}=40\right)$ power functions of the tests $U_{1,1}, U_{3,1}, U_{5,1}, U_{5,3}$ and $A(\hat{S})$; densities: doubleexponential, Cauchy and exponential (Continuation from Figure 3.)
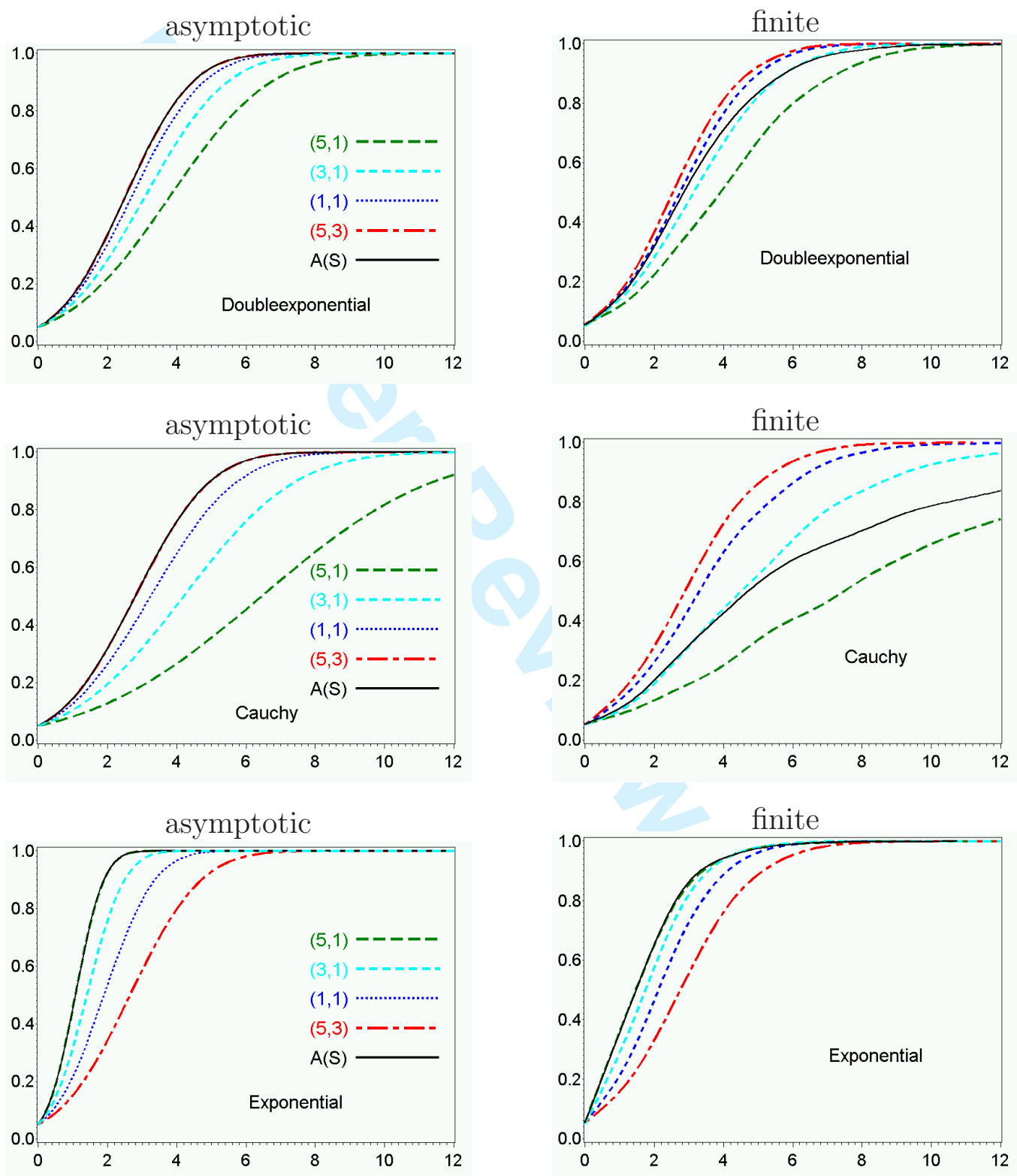
Figure 5. Running times for two age groups

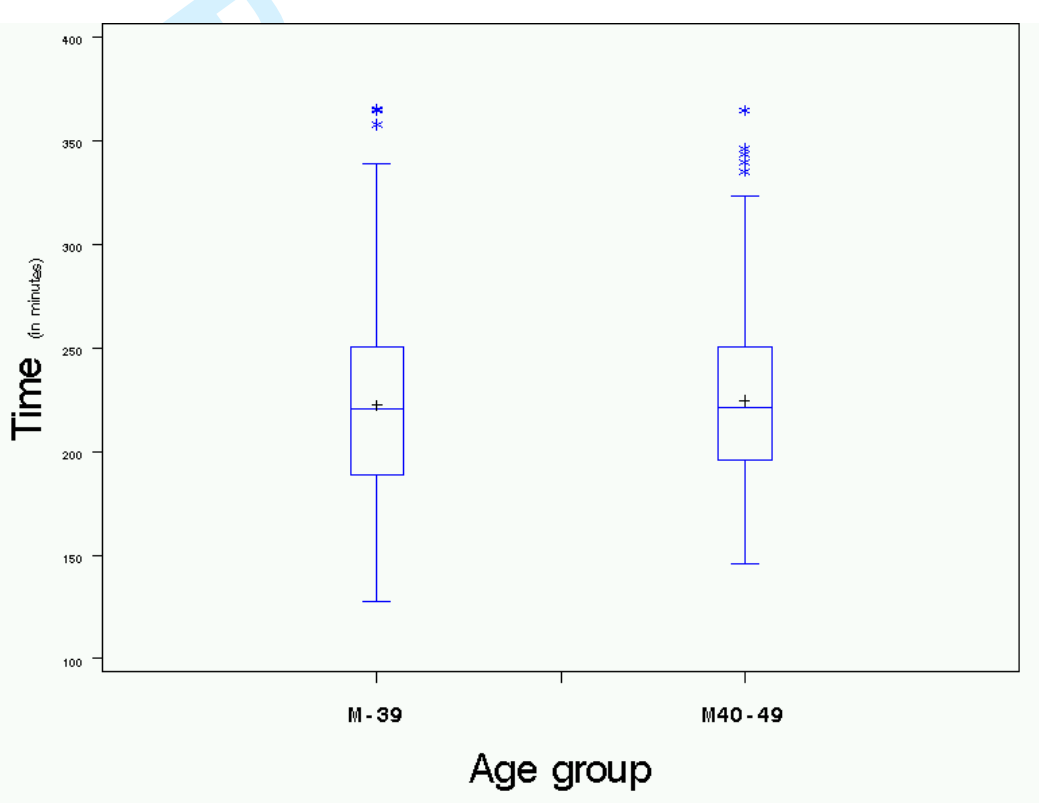


Table 1. Values of the factors $C_{k, i}^{2}(f), k \leq 5$, for various densities $f$, together with the corresponding factors for the $t$-test

\begin{tabular}{|c|c|c|c|c|c|c|c|c|c|c|}
\hline Density & $t$-test & $U_{1,1}$ & $U_{2,1}$ & $U_{3,1}$ & $U_{3,2}$ & $U_{4,1}$ & $U_{4,2}$ & $U_{5,1}$ & $U_{5,2}$ & $U_{5,3}$ \\
\hline Uniform & 12.00 & 12.00 & 14.74 & 22.09 & 7.053 & 30.01 & 7.535 & 38.00 & 9.109 & 6.095 \\
\hline & 1.000 & 0.954 & 0.976 & 0.985 & 0.873 & 0.950 & 0.889 & 0.900 & 0.931 & 0.832 \\
\hline Logistic & 0.304 & 0.333 & 0.332 & .313 & 0.324 & 0.284 & 0.327 & 0.254 & 0.332 & 0.315 \\
\hline Doubleex & 500 & 750 & 0.705 & 576 & 0.833 & 0.462 & 0.816 & 0.379 & 0.749 & 0.866 \\
\hline Cauchy & 0.0 & 04 & 0.268 & 82 & 0.382 & .118 & 0.368 & 0.077 & 0.318 & 409 \\
\hline$t_{2}$ & 0.0 & 0.520 & 0.489 & 398 & 0.572 & 311 & 0.566 & 0.244 & 0.538 & .579 \\
\hline$t_{10}$ & 0.800 & $\underline{0.843}$ & 0.846 & 0.816 & 0.802 & 0.755 & 0.813 & 0.688 & 0.835 & 0.772 \\
\hline L-DE 0.55 & 0.499 & 0.745 & 0.701 & 0.574 & 0.826 & & & & 0.728 & $\underline{0.822}$ \\
\hline & 0.495 & 0.726 & 0.686 & 0.567 & 0.798 & 0.458 & 0.783 & 0.377 & 0.728 & 0.822 \\
\hline & 0 & 0 & 0.641 & 0.544 & 20 & & 12 & 0.373 & 0.678 & 0.730 \\
\hline & & 0. & ( & 0.524 & 0. & & 59 & 68 & 0.637 & 0.665 \\
\hline & & 0.581 & 0.563 & 0.497 & 0.599 & 23 & 99 & 0.360 & 0.587 & $\underline{0.594}$ \\
\hline & & 0.464 & & 0.422 & 0.460 & & & 0.328 & 0.466 & 0.450 \\
\hline & & & 0.396 & & & & & & & .380 \\
\hline & & & & & & & & & & 0.353 \\
\hline & & 0.3 & & & & & & & & 328 \\
\hline & & $\underline{0.298}$ & 0.299 & 39 & 0 & 0 & & 42 & 96 & 271 \\
\hline & & $\overline{0.250}$ & 0.254 & 5 & 0.228 & & 0 . & 0.224 & 48 & 0.215 \\
\hline & & 173 & & & 0.147 & & 0 . & $\underline{0.188}$ & & 0.134 \\
\hline & & 013 & 0.139 & 0.156 & 0.105 & & & $\underline{0.161}$ & & .094 \\
\hline & & & & & & & & & & .061 \\
\hline & & & & & & & & & & 0.015 \\
\hline & & & & & & & & & 44 & 0.060 \\
\hline & & & 0. & & & 0 . & 0 . & 82 & 0.480 & 550 \\
\hline & & & & & & & & & 19 & 1.016 \\
\hline & & 52 & 1.850 & 1.537 & 2.113 & & & & 2.003 & 2.132 \\
\hline & & & & & & & & & & $\underline{5.662}$ \\
\hline & & & 0 & & & & & & & 26.71 \\
\hline & & 148 & 149.2 & 144 & & & & & .0 & 136.7 \\
\hline & & $\overline{23}$ & 23.56 & 3 & & & & 21.59 & 22.50 & 20.19 \\
\hline & & & 13.33 & & & & & 13.20 & 12.45 & 10.91 \\
\hline & & & 5.425 & 6.196 & & 6.686 & 4.222 & 6.978 & 4.643 & 3.762 \\
\hline & & & & & & & & & & 823 \\
\hline & & & & & & & & & & \\
\hline & & & & & & & & & & 0787 \\
\hline & & & & $\overline{0.811}$ & & & & 94 & 0.815 & 0.744 \\
\hline & & & & 0.954 & & & & 0.859 & 0.913 & 0.820 \\
\hline & & 0.914 & 0.928 & $\underline{0.924}$ & & 0.878 & 0.860 & 0.819 & 0.896 & 0.807 \\
\hline & & & 0.861 & $\underline{0.839}$ & & & & 0.713 & 846 & 0.771 \\
\hline & & & & & & & & & & 0.712 \\
\hline & & & & $\underline{0.943}$ & & & & 41 & 0.909 & .817 \\
\hline & & & & 002 & & & & 0.786 & 0.887 & 0.802 \\
\hline & & & & & & & & & & 0.757 \\
\hline $\mathrm{CN}(.05,5)$ & 0.455 & $\underline{0.709}$ & 0.696 & $\overline{0.631}$ & 0.707 & 0.544 & 0.713 & 0.461 & 0.726 & 0.686 \\
\hline
\end{tabular}


Continuation from the previous page

\begin{tabular}{|c|c|c|c|c|c|c|c|c|c|c|}
\hline Density & test & $U_{1,1}$ & $U_{2,1}$ & $U_{3,1}$ & $U_{3,2}$ & $U_{4,1}$ & $U_{4,2}$ & $U_{5,1}$ & $U_{5,2}$ & $U_{5,3}$ \\
\hline Mielke $(0.2)$ & 0.452 & 0.620 & 0.591 & 0.500 & 0.667 & 0.413 & 0.658 & 0.347 & 0.620 & .682 \\
\hline Mielke (0.4) & & 0.521 & 0503 & & 0.545 & 373 & 11 & 319 & 21 & 548 \\
\hline & & $\underline{0.444}$ & & & & & & & & 440 \\
\hline & & $\overline{0.383}$ & 377 & 48 & 380 & 09 & 382 & 273 & & .374 \\
\hline & & 245 & 249 & 246 & 227 & & 231 & 215 & 242 & 0.215 \\
\hline & & 88 & & .200 & 167 & 95 & 171 & 185 & & 0.156 \\
\hline & & 0.061 & 67 & & 047 & & 049 & 90 & 057 & 0.042 \\
\hline & & 6 & & & 04 & & 004 & & 05 & 0.003 \\
\hline & & 0 & & & 540 & 72 & 45 & 20 & 50 & 0.626 \\
\hline D & & 444 & & 389 & 54 & 40 & 552 & 98 & 41 & .454 \\
\hline & & 2 & 9 & 39 & & & & & & 318 \\
\hline BT & & 0.118 & & & & & 32 & 58 & .123 & 136 \\
\hline & & & & & & & & 55 & .080 & 0.081 \\
\hline & & & & & & & & & 277 & 1.524 \\
\hline & & & & & & & & & & 0.808 \\
\hline & & & & & & & & & 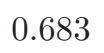 & 0.546 \\
\hline & & & & & & & & & & 0.412 \\
\hline & & & & & & & & & & 330 \\
\hline & & & & & & & & & 78 & 0.237 \\
\hline & & & & & & & & & & 184 \\
\hline & & & & & & & & & & 0.087 \\
\hline & & & & & & & 08 & & & 1.673 \\
\hline & & & & & & & & & & 2.446 \\
\hline & & & & & & & & 470 & 28 & 3.738 \\
\hline & & & & & & & & $\overline{7.182}$ & 16 & 5.364 \\
\hline & & & & & & & & $\overline{74}$ & 87 & 7.315 \\
\hline & & & & & & & & & & 12.18 \\
\hline & & & & & & & & $\underline{21.39}$ & .76 & 18.32 \\
\hline & & & & & & & & .2 & & 332.9 \\
\hline & & & & & & 96.18 & & 91.29 & 3.41 & 83.31 \\
\hline & & & & & & & & & & 9.363 \\
\hline & & & & & & & & & & 3.448 \\
\hline & & & & & & & & & & 1.153 \\
\hline & & & & & & & & & 56 & 0.959 \\
\hline & & & & & & & & & & 0.817 \\
\hline & & & & & & 24 & & & .947 & 0.509 \\
\hline & & & & & & & & & 56 & 5.976 \\
\hline & & & & & & & & & & 5.040 \\
\hline & & & & & & & & & & 3.284 \\
\hline & & & & & & & & & & 2.116 \\
\hline & & & & & & & & & 1.495 & 1.471 \\
\hline $\operatorname{RST}(-.4,-.49)$ & 0.020 & 0.659 & 0.613 & 0.485 & 0.742 & 0.369 & 0.730 & 0.282 & 0.681 & 0.760 \\
\hline
\end{tabular}


Table 2. Values of the tailweight $t$ for various symmetric densities

\begin{tabular}{lc|lc|lc|lc}
\hline Density & $t$ & Density & $t$ & Density & $t$ & Density & $t$ \\
\hline Uniform & 1.286 & Normal & 1.587 & Logistic & 1.697 & Doubleexp & 1.912 \\
Cauchy & 3.217 & $t_{2}$ & 2.107 & $t_{10}$ & 1.672 & & \\
L-DE 0.55 & 1.911 & L-DE 0.61 & 1.905 & L-DE 0.70 & 1.884 & L-DE 0.75 & 1.864 \\
L-DE 0.80 & 1.836 & L-DE 0.90 & 1.753 & L-DE 0.95 & 1.712 & L-DE 0.97 & 1.703 \\
L-DE 0.99 & 1.698 & & & & & & \\
U-L 0.55 & 1.668 & U-L 0.61 & 1.623 & U-L 0.70 & 1.534 & U-L 0.75 & 1.474 \\
U-L 0.80 & 1.409 & U-L 0.90 & 1.300 & & & & \\
RST (-1) & 3.451 & RST (-0.5) & 2.302 & RST (-0.4) & 2.146 & RST (-0.3) & 2.010 \\
RST (-0.2) & 1.891 & RST (-0.1) & 1.788 & RST (0.05) & 1.657 & RST (0.14) & 1.591 \\
RST (0.2) & 1.552 & RST (0.4) & 1.446 & & & & \\
CN (.01,2) & 1.592 & CN (.02,2) & 1.597 & CN (.03,2) & 1.601 & CN (.05,2) & 1.611 \\
CN (.01,3) & 1.596 & CN (.02,3) & 1.605 & CN (.03,3) & 1.615 & CN (.05,3) & 1.635 \\
CN (.01,5) & 1.600 & CN (.02,5) & 1.614 & CN (.03,5) & 1.629 & CN (.05,5) & 1.665 \\
Mielke 0.2 & 1.860 & Mielke 0.4 & 1.812 & Mielke 0.6 & 1.770 & Mielke 0.8 & 1.732 \\
Mielke 1.5 & 1.626 & Mielke 2.0 & 1.571 & Mielke 5.0 & 1.404 & Mielke 20.0 & 1.292 \\
BT (0.25) & 1.670 & BT(0.5) & 1.752 & BT(0.75) & 1.833 & BT (1.25) & 1.992 \\
BT (1.5) & 2.071 & \multicolumn{1}{l|}{} & & & & \\
\hline
\end{tabular}


Table 3. Values of skewness $s$, tailweight $t$, left tailweight $t_{l}$ and right tailweight $t_{r}$ for various skew densities

\begin{tabular}{l|cccc}
\hline Density & Skewness & Tailweight & Left tailweight & Right tailweight \\
\hline Exponential & 0.564 & 1.697 & 1.210 & 1.912 \\
Gamma (1.5) & 0.460 & 1.651 & 1.284 & 1.846 \\
Gamma (2.0) & 0.401 & 1.668 & 1.329 & 1.810 \\
Gamma (2.5) & 0.354 & 1.621 & 1.360 & 1.785 \\
Gamma (3.0) & 0.338 & 1.653 & 1.382 & 1.768 \\
Gamma (4.0) & 0.298 & 1.643 & 1.412 & 1.743 \\
Gamma (5.0) & 0.271 & 1.636 & 1.432 & 1.727 \\
Gamma (10.0) & 0.202 & 1.619 & 1.480 & 1.686 \\
Weibull (1.1) & 0.508 & 1.654 & 1.237 & 1.859 \\
Weibull (1.5) & 0.335 & 1.571 & 1.329 & 1.728 \\
Weibull (2.0) & 0.194 & 1.544 & 1.411 & 1.649 \\
Weibull (2.5) & 0.103 & 1.541 & 1.470 & 1.605 \\
Weibull (3.0) & 0.040 & 1.546 & 1.513 & 1.577 \\
Weibull (4.0) & -0.04 & 1.559 & 1.573 & 1.544 \\
Weibull (5.0) & -0.088 & 1.571 & 1.612 & 1.525 \\
LogNormal (0.05) & 0.041 & 1.588 & 1.563 & 1.611 \\
LogNormal (0.1) & 0.082 & 1.591 & 1.541 & 1.634 \\
LogNormal (0.3) & 0.242 & 1.626 & 1.457 & 1.749 \\
LogNormal (0.5) & 0.390 & 1.695 & 1.386 & 1.879 \\
LogNormal (0.9) & 0.629 & 1.940 & 1.274 & 2.202 \\
LogNormal (1.0) & 0.676 & 2.024 & 1.251 & 2.298 \\
LogNormal (1.1) & 0.719 & 2.117 & 1.229 & 2.401 \\
LogNormal (1.5) & 0.844 & 2.588 & 1.160 & 2.890 \\
RST (0.2,0.4) & 0.029 & 1.486 & 1.486 & 1.486 \\
RST (0.2,0.49) & 0.014 & 1.459 & 1.469 & 1.449 \\
RST (0.4,0.49) & -0.011 & 1.426 & 1.436 & 1.417 \\
RST (-0.2-,0.4) & 0.375 & 2.073 & 1.718 & 2.289 \\
RST (-0.2,-0.49) & 0.490 & 2.195 & 1.657 & 2.470 \\
RST (-0.4,-0.49) & 0.153 & 2.226 & 2.086 & 2.342 \\
\hline
\end{tabular}


Table 4. The factors $C_{k, i}^{2}(f)$, for the chosen $U_{k, i}$-tests, and the corresponding factors for the linear rank tests, for various densities $f$, only the entries that are on the first and second place, respectively

\begin{tabular}{l|rrrr|rrrr}
\hline & \multicolumn{3}{|c}{$A(S)$} & \multicolumn{5}{c}{$B(S)$} \\
\hline density & $U_{1,1}$ & $U_{3,1}$ & $U_{5,1}$ & $U_{5,3}$ & GA & WI & LT & HFR \\
\hline Uniform & & 22.0 & $\mathbf{3 8 . 0}$ & & $\mathbf{2 4 . 0}$ & 12.0 & & \\
Normal & 0.954 & $\mathbf{0 . 9 8 5}$ & & & 0.884 & $\mathbf{0 . 9 5 4}$ & & \\
Logistic & $\mathbf{0 . 3 3 3}$ & & & 0.315 & & $\mathbf{0 . 3 3 3}$ & 0.315 & \\
Doubleexpon & 0.750 & & & $\mathbf{0 . 8 6 6}$ & & 0.750 & $\mathbf{0 . 8 4 5}$ & \\
Cauchy & 0.304 & & & $\mathbf{0 . 4 0 9}$ & & 0.304 & $\mathbf{0 . 4 0 7}$ & \\
Exponential & & 5.52 & $\mathbf{9 . 5 0}$ & & $\mathbf{6 . 0 0}$ & & & 5.40 \\
\hline U-L (0.75) & 0.130 & & $\mathbf{0 . 1 6 1}$ & & $\mathbf{0 . 1 6 6}$ & 0.130 & & \\
L-D (0.75) & 0.630 & & & $\mathbf{0 . 6 6 5}$ & & 0.630 & $\mathbf{0 . 6 6 6}$ & \\
\hline
\end{tabular}


Table 5. Rankings of the $t$-test $(t)$, the Wilcoxon test (W), and the adaptive test (A)

\begin{tabular}{|c|c|c|c|c|c|c|c|c|c|c|c|}
\hline Density & $t$ & $\bar{W}$ & $\mathrm{~A}$ & Density & $t$ & W & $\mathrm{A}$ & Density & $t$ & $\mathrm{~W}$ & $\mathrm{~A}$ \\
\hline Uniform & 2.5 & 2.5 & 1 & RST $(0.05)$ & 3 & 1.5 & 1.5 & Exponential & 3 & 2 & 1 \\
\hline Normal & 1 & 3 & 2 & RST (0.14) & 1 & 3 & 2 & Gamma (1.5) & 3 & 2 & 1 \\
\hline Logistic & 3 & 1.5 & 1.5 & $\operatorname{RST}(0.2)$ & 1 & 3 & 2 & Gamma (2.0) & 3 & 2 & 1 \\
\hline Doubleex & 3 & 2 & 1 & $\operatorname{RST}(0.4)$ & 2 & 3 & 1 & Gamma (2.5) & 3 & 2 & 1 \\
\hline Cauchy & 3 & 2 & 1 & $\mathrm{CN}(.01,2)$ & 1 & 3 & 2 & Gamma (3.0) & 3 & 2 & 1 \\
\hline$t_{2}$ & 3 & 2 & 1 & $\mathrm{CN}(.02,2)$ & 2 & 3 & 1 & Gamma (4.0) & 3 & 2 & 1 \\
\hline$t_{10}$ & 3 & 1.5 & 1.5 & $\mathrm{CN}(.03,2)$ & 1 & 3 & 2 & Gamma (5.0) & 3 & 2 & 1 \\
\hline L-DE 0.55 & 3 & 2 & 1 & $\mathrm{CN}(.05,2)$ & 1 & 3 & 2 & Gamma (10) & 3 & 2 & 1 \\
\hline L-DE 0.61 & 3 & 2 & 1 & $\mathrm{CN}(.01,3)$ & 3 & 2 & 1 & Weibull (1.1) & 3 & 2 & 1 \\
\hline L-DE 0.70 & 3 & 2 & 1 & $\mathrm{CN}(.02,3)$ & 3 & 2 & 1 & Weibull (1.5) & 3 & 2 & 1 \\
\hline L-DE 0.75 & 3 & 2 & 1 & $\mathrm{CN}(.03,3)$ & 3 & 1 & 2 & Weibull (2.0) & 3 & 2 & 1 \\
\hline L-DE 0.80 & 3 & 2 & 1 & $\mathrm{CN}(.05,3)$ & 3 & 1 & 2 & Weibull (2.5) & 2 & 3 & 1 \\
\hline L-DE 0.90 & 3 & 1.5 & 1.5 & $\mathrm{CN}(.01,5)$ & 3 & 2 & 1 & Weibull (3.0) & 1 & 3 & 2 \\
\hline L-DE 0.95 & 3 & 1.5 & 1.5 & $\mathrm{CN}(.02,5)$ & 3 & 2 & 1 & Weibull (4.0) & 1 & 3 & 2 \\
\hline L-DE 0.97 & 3 & 1.5 & 1.5 & $\mathrm{CN}(.03,5)$ & 3 & 1 & 2 & Weibull (5.0) & 2 & 3 & 1 \\
\hline L-DE 0.99 & 3 & 1.5 & 1.5 & $\mathrm{CN}(.05,5)$ & 3 & 1.5 & 1.5 & LogNor $(0.05)$ & 1 & 3 & 2 \\
\hline U-L 0.55 & 3 & 1.5 & 1.5 & Mielke (0.2) & 3 & 2 & 1 & LogNor $(0.1)$ & 2 & 3 & 1 \\
\hline U-L 0.61 & 3 & 1.5 & 1.5 & Mielke (0.4) & 3 & 2 & 1 & LogNor $(0.3)$ & 3 & 2 & 1 \\
\hline U-L 0.70 & 3 & 2 & 1 & Mielke (0.6) & 3 & 1.5 & 1.5 & LogNor $(0.5)$ & 3 & 2 & 1 \\
\hline U-L 0.75 & 3 & 2 & 1 & Mielke (0.8) & 3 & 1.5 & 1.5 & LogNor $(0.9)$ & 3 & 2 & 1 \\
\hline U-L 0.80 & 3 & 2 & 1 & Mielke (1.5) & 3 & 2 & 1 & LogNor (1.0) & 3 & 2 & 1 \\
\hline U-L 0.90 & 3 & 2 & 1 & Mielke (2.0) & 2 & 3 & 1 & LogNor (1.1) & 3 & 2 & 1 \\
\hline $\operatorname{RST}(-1)$ & 3 & 2 & 1 & Mielke (5.0) & 2 & 3 & 1 & LogNor (1.5) & 3 & 2 & 1 \\
\hline $\operatorname{RST}(-0.5)$ & 3 & 2 & 1 & Mielke (20) & 3 & 2 & 1 & $\operatorname{RST}(.2, .4)$ & 2 & 3 & 1 \\
\hline $\operatorname{RST}(-0.4)$ & 3 & 2 & 1 & $\mathrm{BT}(0.25)$ & 3 & 1.5 & 1.5 & $\operatorname{RST}(.2, .49)$ & 2 & 3 & 1 \\
\hline $\operatorname{RST}(-0.3)$ & 3 & 2 & 1 & $\mathrm{BT}(0.5)$ & 3 & 1.5 & 1.5 & $\operatorname{RST}(.4 ., 49)$ & 2 & 3 & 1 \\
\hline $\operatorname{RST}(-0.2)$ & 3 & 2 & 1 & $\mathrm{BT}(0.75)$ & 3 & 2 & 1 & $\operatorname{RST}(-.2,-.4)$ & 3 & 2 & 1 \\
\hline \multirow[t]{2}{*}{$\operatorname{RST}(-0.1)$} & 3 & 1.5 & 1.5 & $\mathrm{BT}(1.25)$ & 3 & 2 & 1 & $\operatorname{RST}(-.2,-.49)$ & 3 & 2 & 1 \\
\hline & & & & $\mathrm{BT}(1.5)$ & 3 & 2 & 1 & $\operatorname{RST}(-.4,-.49)$ & 3 & 2 & 1 \\
\hline
\end{tabular}




\section{Rejoinder to the second report of referee B}

Thank you very much for your report on our revised paper. All your comments are considered.

In a new section (Section 6) we compare the adaptive test with the Wilcoxon test and with the $t$-test. We think, the improvement of the asymptotic power is convincing.

You are right, the restriction $k \leq 5$ looks slightly restrictive, and the computer time argument is not satisfactory. However, we have to make some restriction. We think, the arguments presented in the first paragraph of page 5 are sufficient.

We have changed "generalized" and "generalization" to "generalised" and "generalisation". We are sorry, we cannot see any other American English words. The text is thought to be written in British English.

In Table 1 we added the asymptotic efficacies of the $t$-test. We prefer to present the asymptotic efficacies instead of some ARE for the following reasons:

First, the paper is on U-statistics, and if any test is chosen to be the reference test for computing AREs, then it should be the Wilcoxon test. Second, there are some densities for which the AE of the t-test is zero, all ARE-values in the corresponding row become $\infty$. Third, the AE allow easier comparisons. The reader may take his/her favourite test and compare it to the others.

We decided to add no further curves in Figures 3 and 4. One reason is that there are already five lines, and a sixth one will reduce the readability of the curves. Let us illustrate this fact: For the uniform the two lines for the $t$-test and $U_{1,1}$ coincide, for the normal the line for the $t$-test lies very slightly above the other lines, but this will not be visible. For the logistic the line for the $t$-tests is almost the same as for the tests $U_{3,1}$ and $U_{5,3}$, and for the Cauchy no line exists. An impression of the asymptotic power of the $t$-test can be obtained from the corresponding entries in Table 1. Another point is that the $t$-test plays only a marginal role in our paper.

We used the SAS package to draw the figures. Concerning the blue dotted and dashed lines this program seems to produce some problems. We are currently working on it. However, we are not able to do it in the narrow time window of two weeks that we have for revision.

In Figure 1 we labelled the dot in the upper left corner. Labels on the axes are removed, since an detailled interpretation of the axes is difficult.

The other mistakes are corrected. 
Figure 3. The asymptotic and finite $\left(n_{1}=n_{2}=40\right)$ power functions of the tests $U_{1,1}, U_{3,1}, U_{5,1}, U_{5,3}$ and $A(\hat{S})$; densities: uniform, normal and logistic.
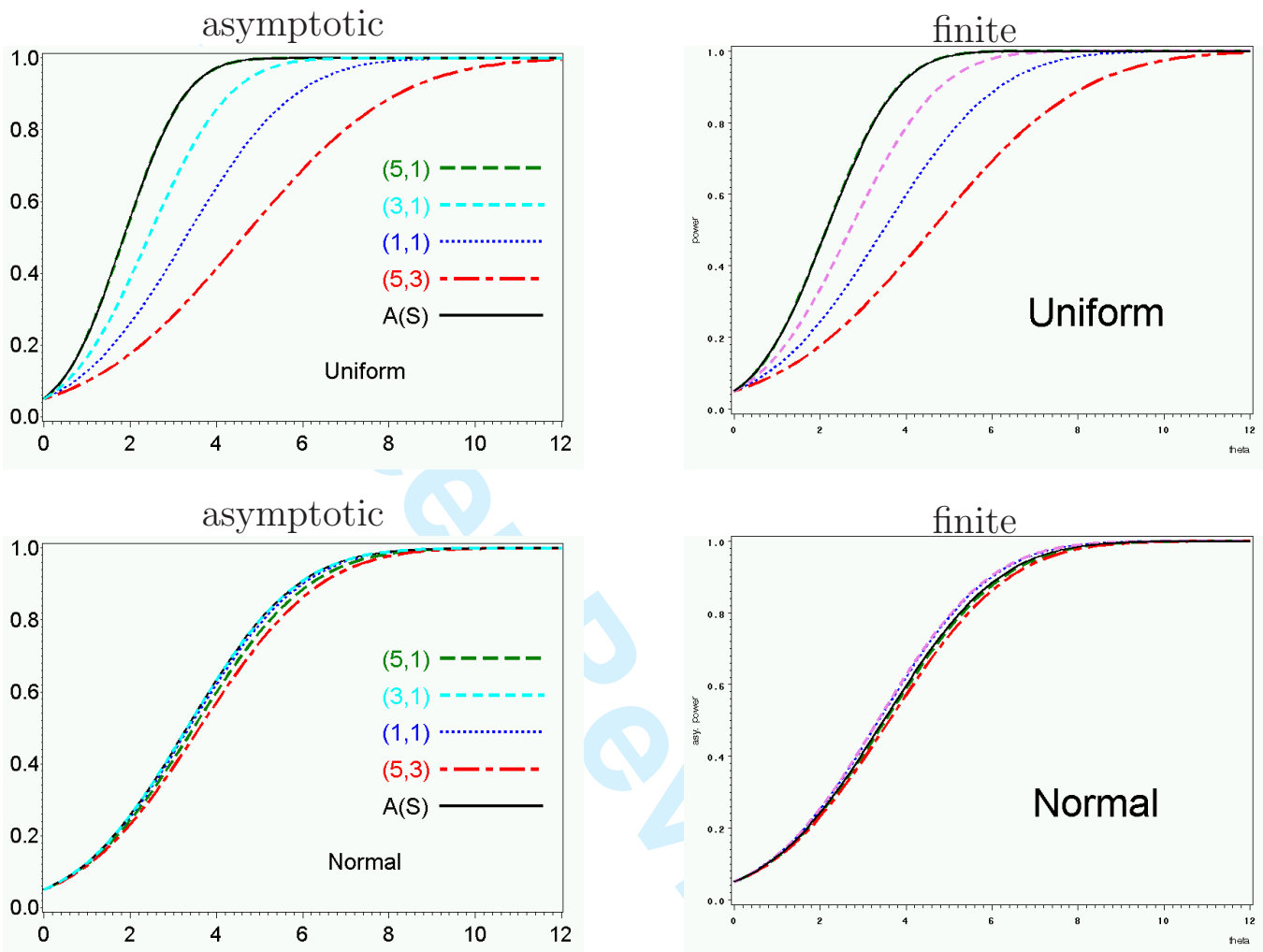

asymptotic
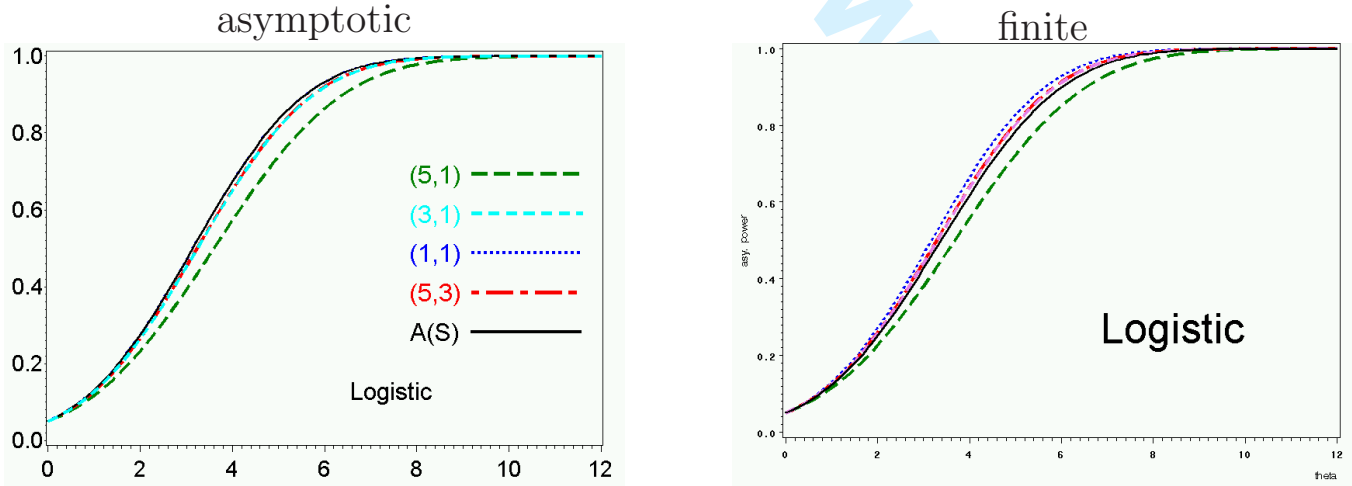
Figure 4. The asymptotic and finite $\left(n_{1}=n_{2}=40\right)$ power functions of the tests $U_{1,1}, U_{3,1}, U_{5,1}, U_{5,3}$ and $A(\hat{S})$; densities: doubleexponential, Cauchy and exponential (Continuation from Figure 3.)
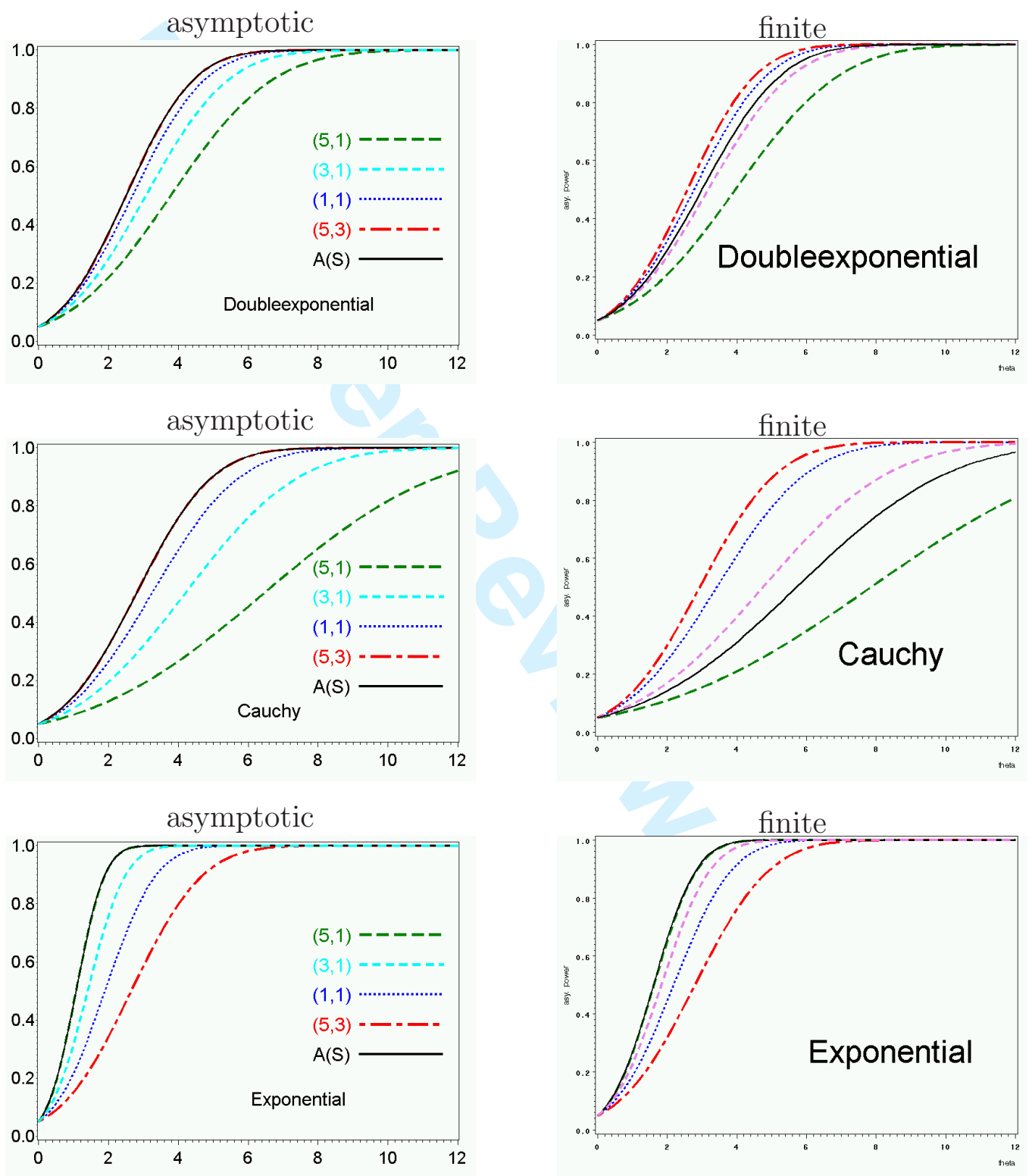